Supporting Information for:

\title{
Evidence for Two Timescale-Specific Blinking Mechanisms in Room-Temperature Single Nanoplatelets
}

Shawn Irgen-Gioro ${ }^{1}$, Yue Wu${ }^{1}$, Rafael López-Arteaga, Suyog Padgaonkar, Jack N. Olding, and Emily A. Weiss*

${ }^{1}$ These Authors contributed equally to this work

Department of Chemistry, Northwestern University, 2145 Sheridan Rd., Evanston, IL 60208-3113

*corresponding author. Email: e-weiss@northwestern.edu

Nanoplatelet Synthesis. The CdSe NPLs and CdSe/CdS core/shell NPLs were synthesized following published procedures with minor modifications. ${ }^{1-3}$ Transmission electron microscope (TEM) images for the 4ML core-only NPLs (Figure S1) and 4ML core-shell NPLs (Figure S2) and corresponding size distributions are shown below.
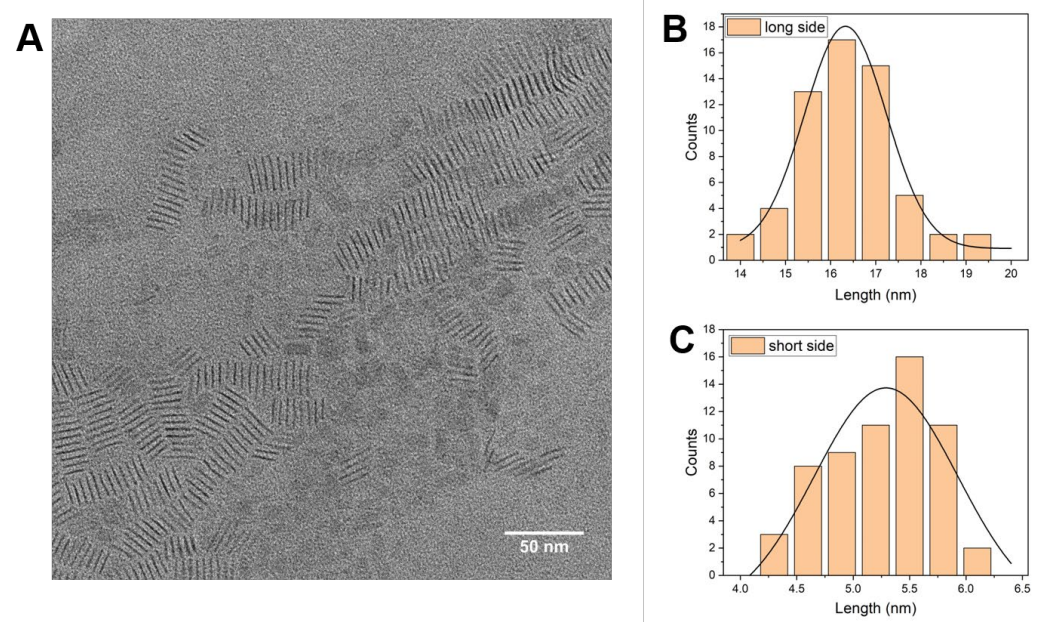

Figure S1. (A) TEM image of 4ML CdSe core-only NPLs. (B,C) Histograms of the lengths of the long side and short side of core-shell NPLs yield an average size of $(16.3 \pm 0.9) \times(5.3 \pm 0.6) \mathrm{nm}$. 

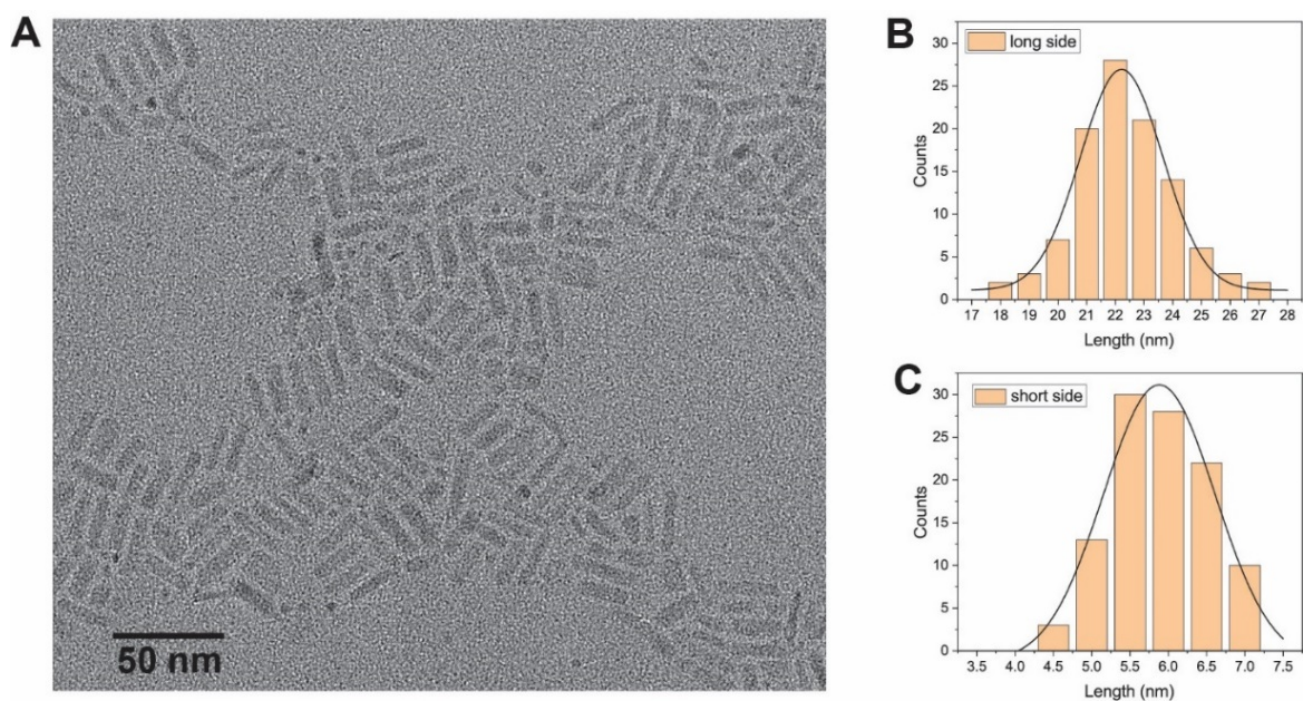

Figure S2. (A) TEM image of $4 \mathrm{ML} / 1 \mathrm{ML} \mathrm{CdSe} / \mathrm{CdS}$ core/shell NPLs. (B,C) Histograms of the lengths of the long side and short side of core-shell NPLs yield an average size of $(22.2 \pm 1.7) \times$ $(5.9 \pm 0.8) \mathrm{nm}$.

Representative Single-Particle Spectra and Blinking Trajectories. An exemplary spatial image of emitters is shown in Figure S3. The dilution of the film is such that emitters are separated by at least a few microns, on average. The resulting emission can be directed into a spectrograph to examine the time-averaged emission spectra, as shown in Figure S3, or, emission spectra can be taken sequentially to obtain successive spectra used to observe spectral diffusion on a $>\sim 100$ ms timescale. The emission of spatially separated emitters can also be sent to APDs resulting in a stream of photons that can be converted into a time trace like Figure S4, and this data can be compiled into count rate histograms as in Figure S5. For the representative data shown in this figure, the core-only sample spends $35 \%$ of the time in the "on" state while the core-shell is $98 \%$ "on". 

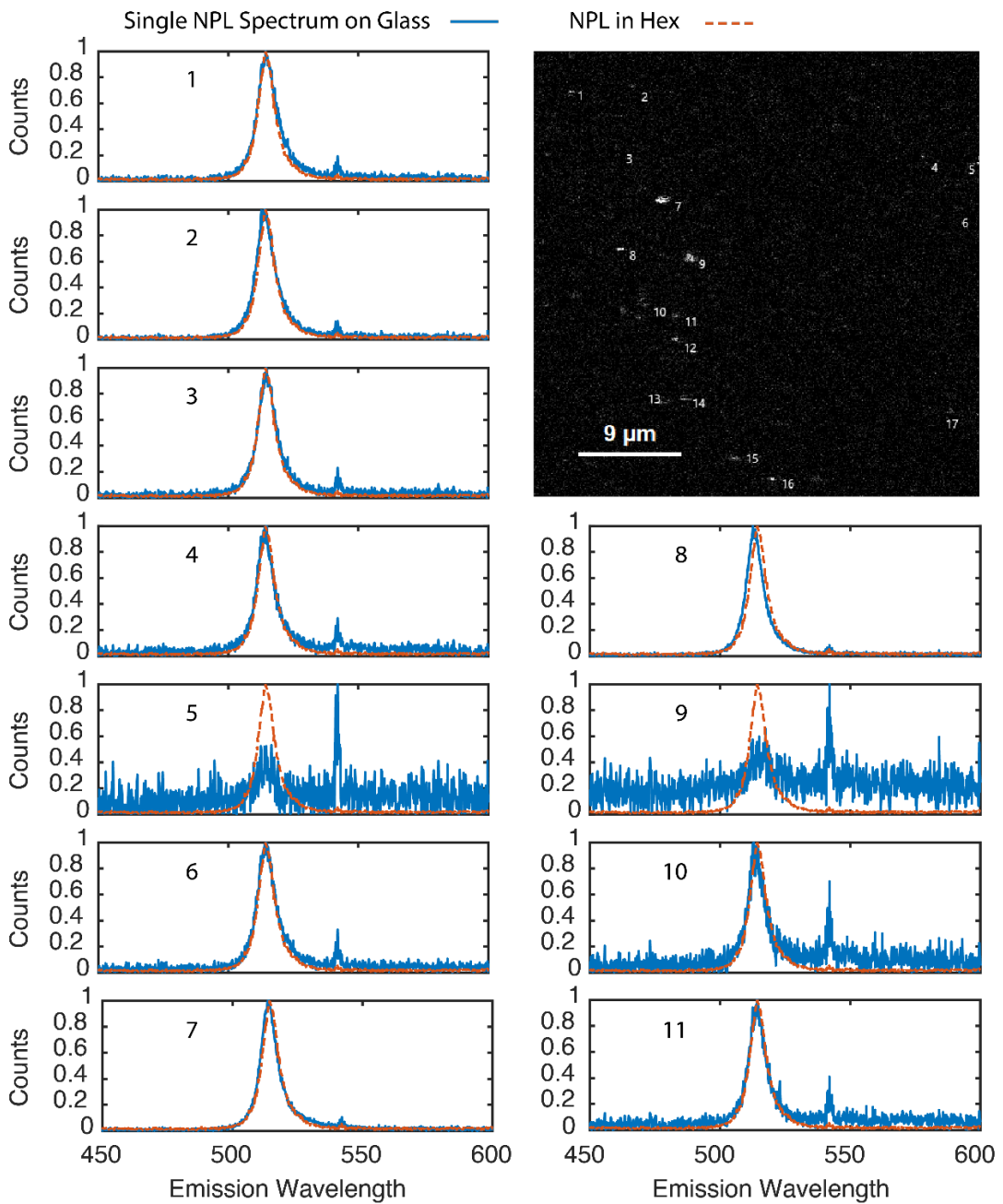

Figure S3. A spatial image of individual 4ML CdSe NPL emitters. The spectrum from each numbered location is displayed, along with the emission spectra of the ensemble of NPLs dispersed in hexane (for comparison). The small peak at $540 \mathrm{~nm}$ in the emission spectra is background light. 

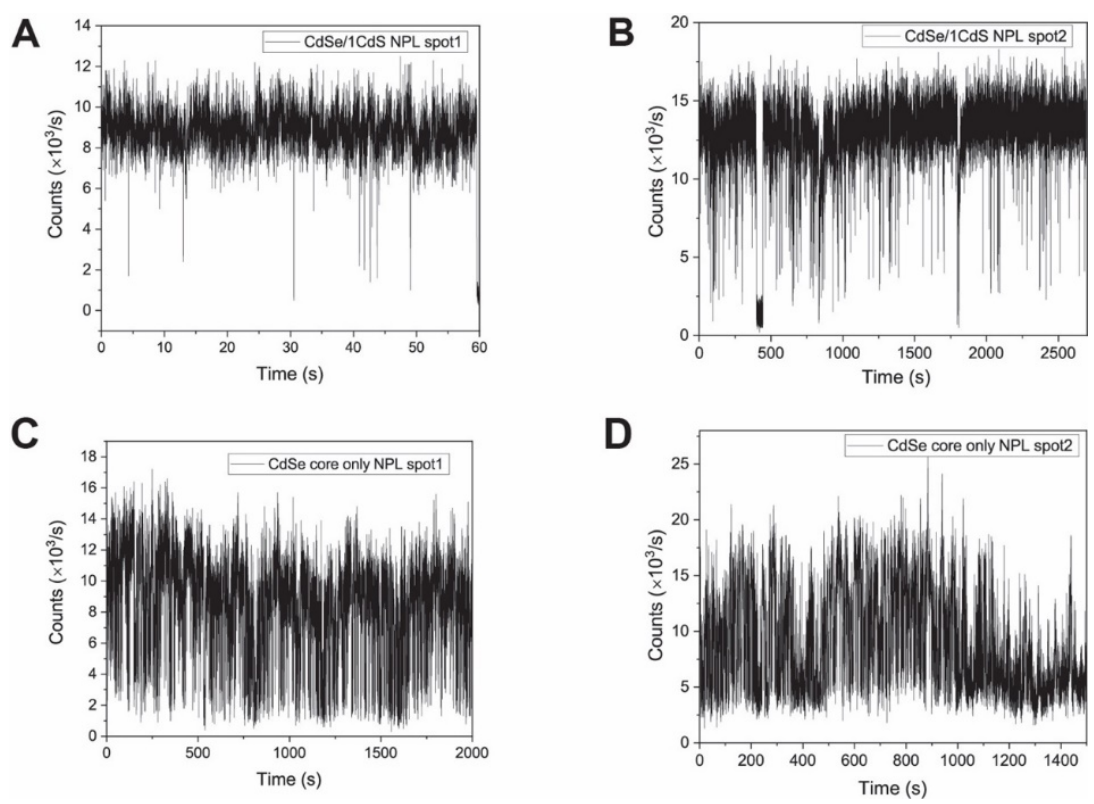

Figure S4. Time traces obtained from two separate 4ML/1ML CdSe/CdS core/shell NPLs (A/B), and two separate 4ML CdSe NPLs (C/D).
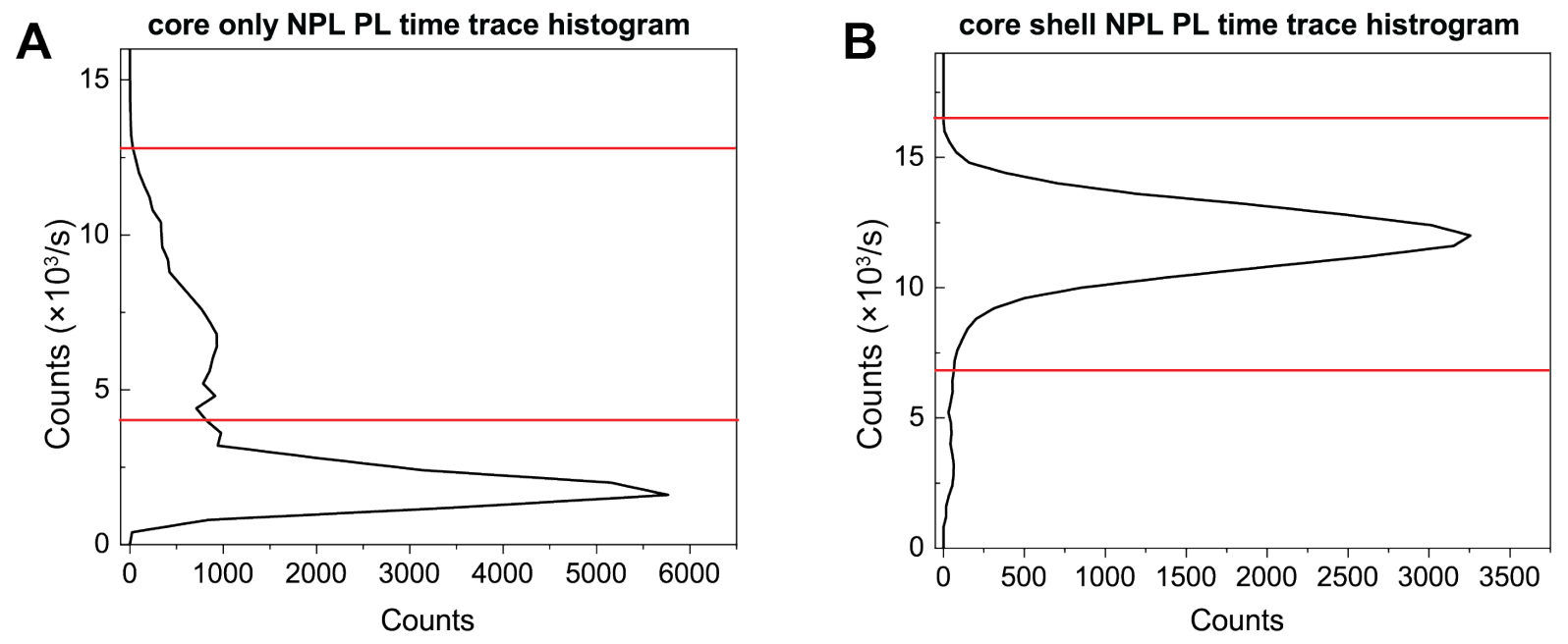

Figure S5. Histograms of the emission count rate for a 4ML core-only CdSe NPL (A), constructed from the data in Figure S4B; and a 4ML/1ML CdSe/CdS core/shell NPL (B), constructed from the data in Figure S4C. The bin size is 400 counts per second. The "on" time percentages (35\% for the core-only NPLs and 98\% for the core/shell NPLs) are determined by integrating the region between red lines divided by the total number time bins. 
Taking the photon streams from the two APDs, we cross-correlated the counts detected to generate an anti-bunching plot (Figure S6). Once we have determined we are indeed looking at a single photon emitter through a confirmation of $g^{(2)}(\tau=0)<0.5$, we proceed to monitoring spectral diffusion.
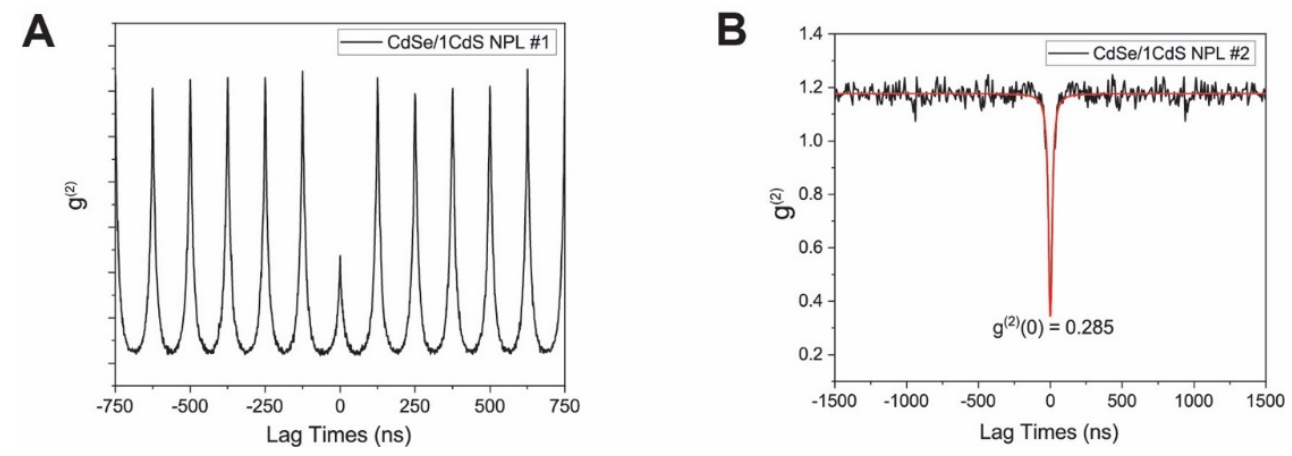

Figure S6. Examples of antibunching plots for single 4ML/1ML CdSe/CdS NPLs using pulsed laser excitation (A), and CW laser excitation (B).

Fitting of the Time-Correlated Single Photon Counting Time Traces. Intensity time traces, like that in Figure 2B of the main text, also contain the timing of photon arrival relative to the excitation pulse, allowing us to create a Time-Correlated Single Photon Counting (TCSPC) trace for each time point. The traces are fit with single exponential functions to extract the lifetimes of the states emitting each photon. The lifetime is then plotted against the emission intensity to generate the plot seen in Figure 2C of the main text.

Figure S7 is a reproduction of Figure $2 \mathrm{C}$ from the main text, where three regions are highlighted: $\mathrm{x}=18-27 \mathrm{~ns}, \mathrm{y}=750-1250$ counts for Region I, $\mathrm{x}=15-25 \mathrm{~ns}, \mathrm{y}=280-400$ counts for region II, and $x=9-16 \mathrm{~ns}, \mathrm{y}=350-650$ counts for region III. From each region, three TCSPC traces are chosen at random and plotted below along with the single exponential fits. 

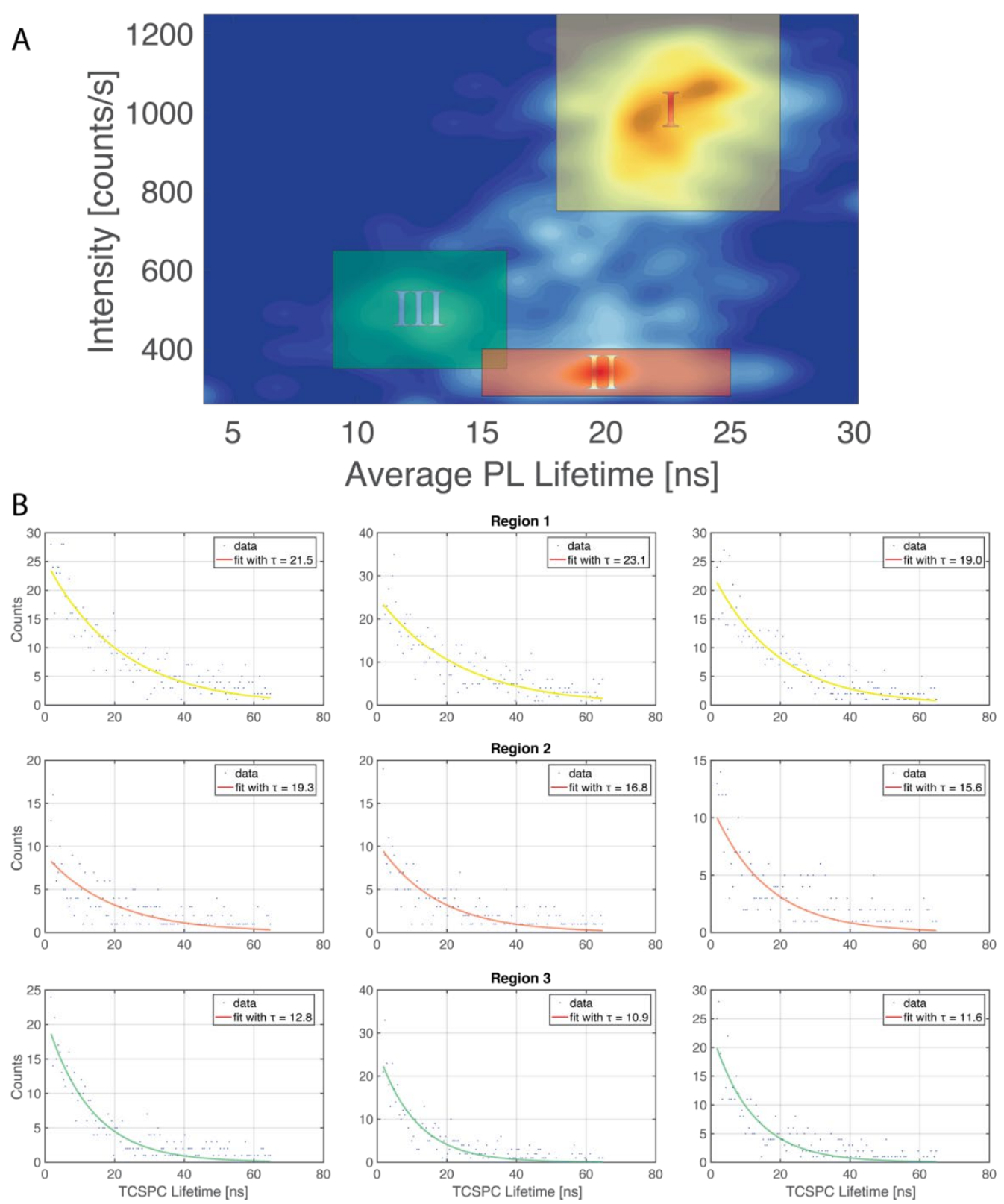

Figure S7. (A) Recreation of Figure 2C of the main text with 3 regions highlighted. (B) Representative traces from each region with the single exponential fit functions used to extract the PL lifetimes.

Correlation Analysis of Sequential Emission Spectra. Observation of spectral diffusion in the spectrometer is most simply done by taking sequential spectra of emitters. Sequential spectra like the one shown in Figure $\mathbf{3 A}$ of the main text can be analyzed to obtain a characteristic timescale of spectral diffusion. This is done through the use of spectral correlations. 
If we assume the spectrum of the emitter $s_{\text {eff }}(\omega)$ is a Gaussian peak with linewidth $\sigma_{e f f}(\tau)$ evolving with correlation time $\tau$,

$$
S_{e f f}(\omega)=\frac{1}{\sigma_{e f f} \sqrt{2 \pi}} e^{-\frac{\left(\omega-\omega_{0}\right)^{2}}{2 \sigma_{e f f}^{2}}}
$$

then the spectral correlation function $p(\zeta, \tau)$ can be written as in eqs $\mathrm{S} 2$.

$$
\begin{aligned}
p(\zeta, \tau) & =\int_{-\infty}^{\infty} s(\omega) s(\omega+\zeta) d \omega \\
& =\int_{-\infty}^{\infty} \frac{1}{\sigma_{e f f} \sqrt{2 \pi}} e^{-\frac{\left(\omega-\omega_{0}\right)^{2}}{2 \sigma_{e f f}^{2}}} \frac{1}{\sigma_{e f f} \sqrt{2 \pi}} e^{-\frac{\left(\omega+\zeta-\omega_{0}\right)^{2}}{2 \sigma_{e f f}^{2}}} d \omega \\
& =\frac{1}{2 \sigma_{e f f}^{2} \pi} \int_{-\infty}^{\infty} e^{-\frac{\left(2 \omega^{2}+2 \omega_{0}^{2}-4 \omega_{0} \omega+2 \zeta \omega-2 \zeta \omega_{0}+\zeta^{2}\right)}{2 \sigma_{e f f}^{2}}} d \omega \\
& =\frac{1}{2 \sigma_{e f f}^{2} \pi} \int_{-\infty}^{\infty} e^{-\frac{\left(2\left(\omega-\omega_{0}+\zeta / 2\right)^{2}+\zeta^{2} / 2\right)}{2 \sigma_{e f f}^{2}}} d \omega \\
& =\frac{1}{2 \sigma_{e f f}^{2} \pi} e^{-\frac{\zeta^{2}}{2\left(\sqrt{2} \sigma_{e f f}\right)^{2}} \int_{-\infty}^{\infty} e^{-\frac{\left(2\left(\omega-\omega_{0}+\zeta / 2\right)^{2}\right)}{2 \sigma_{e f f}^{2}}} d \omega} \\
& =\frac{1}{2 \sigma_{e f f} \sqrt{\pi}} e^{-\frac{\zeta^{2}}{2\left(\sqrt{2} \sigma_{e f f}\right)^{2}}}(\mathrm{~S} 2)
\end{aligned}
$$

The spectral correlation function of a Gaussian spectrum is also a Gaussian, but centered at $\zeta=$ 0 and with a linewidth of $\sqrt{2} \sigma_{e f f}$.

We first calculated the spectral correlation function from the data of Figure 3A. By fitting the spectral correlation function with Gaussian peak function and using the relationship of $\sqrt{2} \sigma_{\text {eff }}$ with $\sigma_{e f f}$, we plot the effective linewidth $\sigma_{e f f}$ vs correlation time $\tau$ in Figure 3C.

Averaging Spectrally Biased Cross-correlation Traces. We used multiple spectrally biased cross-correlation traces similar to Figure 4D of the main text in order to identify the transition between SD-free blinking at $<1 \mathrm{~s}$ timescales and SD-correlated blinking at $>1 \mathrm{~s}$. We did not simply average these traces from separate experiments, since each blinking trajectory results in a different correlation trace. Instead, we averaged the ratio between the cross-correlation and the autocorrelation at each time point. With no $\mathrm{SD}$, the cross-correlation is identical to the autocorrelation and the ratio of the two is 1 . SD introduces anti-correlation in the cross-correlation, 
so, with SD present, the ratio dips below 1. Figure S8 shows the average of seven crosscorrelation:autocorrelation ratios from separate experiments plus the standard deviation from the average as an area plot. The raw individual traces are shown as smaller plots below the main plot. As shown in Figure 4D of the main text, the ratio is within one standard deviation of 1 until $\sim 3 \mathrm{~s}$, when it dips below 1 .
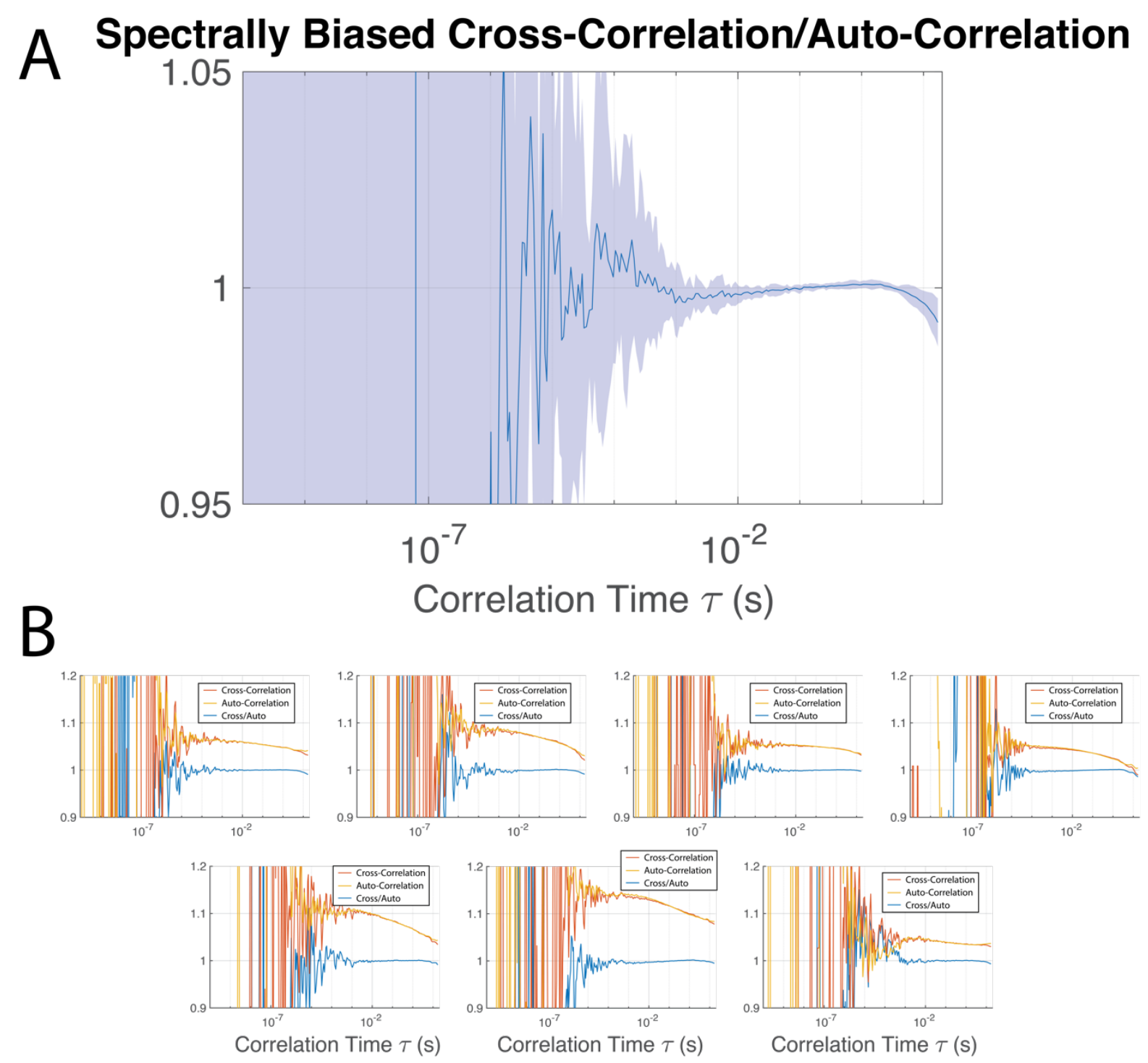

Figure S8. Additional Spectrally Biased Cross Correlation Traces. The average and standard deviation of 7 different spectrally biased cross-correlation/auto-correlation ratios (A). Individual traces of spectrally biased cross-correlations and auto-correlations (B). The ratio of cross/auto correlation is used to average the different traces and not the correlations directly, which vary depending on blinking behavior. 
Photon-Correlated Fourier Spectroscopy (PCFS). The details of the PCFS technique have been extensively documented by the Bawendi group. ${ }^{4-8}$ After photoluminescence is collected from our homebuilt confocal microscope, it is directed to a Michaelson interferometer, which consists of a 2" non-polarizing beam splitter (Newport 20BC17MB.1) and two corner cube retroreflectors (PLX OW-25-1D) mounted on two computer-controlled stages. The corner cube used to dither around a setpoint was a closed-loop piezo stage (Thorlabs NFLDP20S controlled by BPC301). The closed loop functionality allowed us to introduce a sawtooth waveform with a function generator to guarantee a consistent dither movement over two interference fringes at $0.5 \mathrm{~Hz}$. The coarse stage controlling $\delta$ was a Newport SMC100. The output of the interferometer was coupled into two APDs (Micro-Photon Devices PDM) using achromatic lenses (Thorlabs AL1225M-B) mounted in a XYZ translation mount (Thorlabs CXYZ05). The outputs of the APDs were connected to timing electronics that recorded every photon arrival time (Picoquant Picoharp 300). All hardware control was written using Labview with supplied DLLs and data processing was done in Matlab. The log-spaced correlation was calculated using an open source function available online. ${ }^{9}$ The spectrally biased cross-correlation measurements were conducted inside the same interferometer, with the SMC100 stage moved to $\delta=5$ ps so that there was no contribution from interference. Color glass long-pass and short-pass filters were placed in front of the two detectors. All subsequent optics, electronics, and processing was performed similar to the PCFS measurement.

Representative data from the PCFS are shown in Figure S9. Figures S9A,B are the intensity time traces for the two detectors (detector 1 in orange and detector 2 in blue) with a bin size of 100 ms. The traces in Figures S9A,C result from photon streams exhibiting interference, and the traces in Figures S9B,D result from photon streams with no interference. When interference is present, the intensity time trace shows oscillatory behavior as introduced by the stage dither (A), and the cross-correlation falls below the auto-correlation $(\mathbf{C})$. When no interference is present, the intensity on the two detectors look the same, as the stage dither has no detectable effect (B). The lack of interference results in overlapped auto-correlation and cross-correlation traces (D). The use of correlations allows for an extraction of the amplitude of interference for photons separated by a certain lag time $\tau$.

The sensitivity of PCFS is limited at slow timescales due to the timescale on which the optical interference is generated by a stage dither. The stage dither causes the cross-correlation at long 
timescales to oscillate so that a comparison of cross-correlation and auto-correlation is no longer valid. Slowing down the dither can improve this sensitivity, but it introduces additional issues such as microscope drift and sample degradation, because the experiment takes longer to complete. At fast timescales, PCFS is limited due to artifacts of afterpulsing of the APDs. APDs have a finite probability of registering another event after a photon arrives at the APD, so that in the autocorrelation there is a higher probability of seeing another "photon" $\sim 1 \mathrm{~ns}$ after the actual photon arrives. This is not an issue for the cross-correlation, since on the $1 \mathrm{~ns}$ timescale, a photon arrival on detector 1 does not change the probability of a photon arriving on detector 2. In Figure S9(C,D), the timescales at which PCFS are limited are shaded orange. We do not interpret data in these regions.
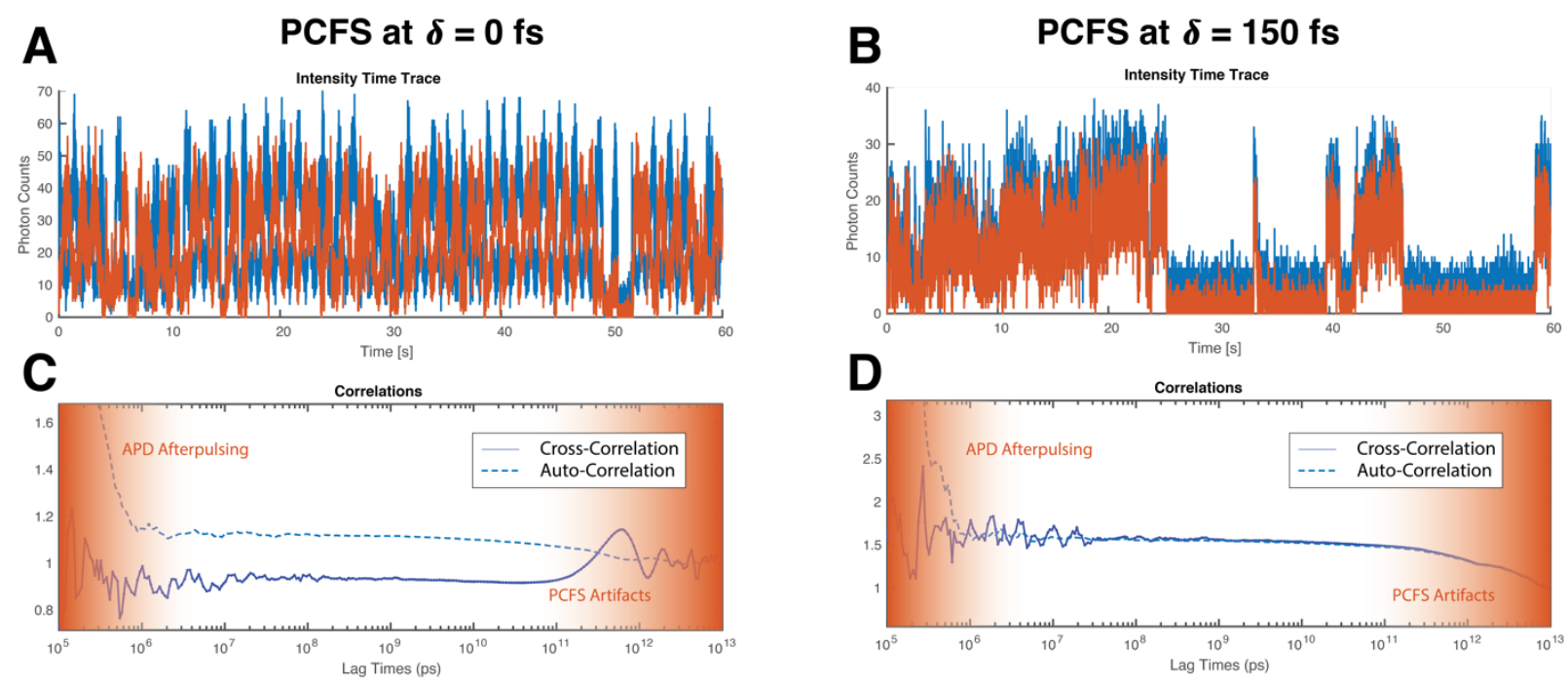

Figure S9. Example of photons exhibiting interference $(\mathbf{A}, \mathbf{C})$ and no interference $(\mathbf{B}, \mathbf{D})$. The intensity time traces are shown for the two detectors with a bin size of $100 \mathrm{~ms}$, for detector 1 in orange and detector 2 in blue. When interference is present, the intensity time trace shows oscillatory behavior as introduced by the stage dither (A), and the cross-correlation falls below the auto-correlation $(\mathbf{C})$. When no interference is present, the intensity on the two detectors look the same, as the stage dither has no detectable effect (B). The lack of interference results in overlapped auto-correlation and cross-correlations traces (D). The orange shaded regions indicate timescales at which PCFS is not sensitive due to either APD afterpulsing or artifacts. 
Statistical Analysis of PCFS Traces. Figure S10 shows 20 additional plots of coherence lifetime $\left(\mathrm{T}_{2}\right) v$ s. correlation time for single $\mathrm{CdSe} / \mathrm{CdS}$ core/shell NPLs. The blue line is the average of these parameters for the 20 samples and the blue shaded region represents one standard deviation from the mean; the raw traces are shown below the average plot. On the timescales at which PCFS is sufficiently sensitive ( $1 \mathrm{~ns}-100 \mathrm{~ms}$ ), no change in $\mathrm{T}_{2}$ is observed with correlation time, indicating a lack of SD on those timescales. 

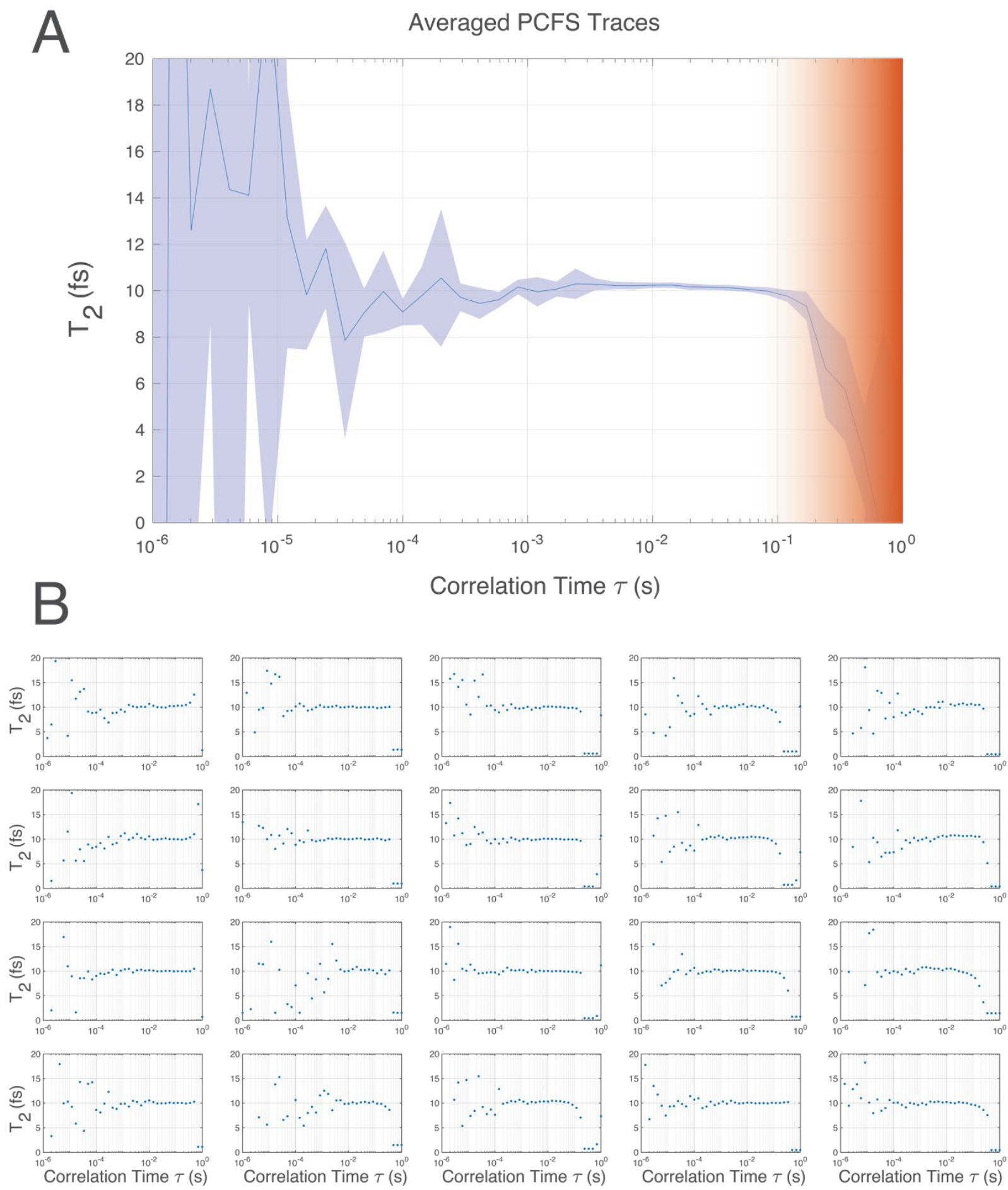

Figure S10. A) Average of the coherence lifetimes $\left(T_{2}\right)$ of 20 different particles (with individual traces collected in (B)) extracted from PCFS data. The orange region is the timescale at which the stage dither limits the sensitivity of PCFS, so we cannot interpret data in this region. Otherwise, $\mathrm{T}_{2}$ is constant within one standard deviation of the mean as a function correlation time, which 
indicates no SD occurs on that timescale. The noise between correlation times of $10^{-6}$ and $10^{-4} \mathrm{~s}$ is present in some, but not all, individual traces and is caused by low count rates. Inspection of the individual traces in (B) with lower noise in that region show that $\mathrm{T}_{2}$ is indeed constant down to a correlation time of at least $10^{-5} \mathrm{~s}$.

\section{REFERENCES}

1. Ithurria, S.; Tessier, M. D.; Mahler, B.; Lobo, R.; Dubertret, B.; Efros, A., Colloidal nanoplatelets with two-dimensional electronic structure. Nat. Mater. 2011, 10 (12), 936-941. 2. Ithurria, S.; Talapin, D. V., Colloidal Atomic Layer Deposition (c-ALD) using Self-Limiting Reactions at Nanocrystal Surface Coupled to Phase Transfer between Polar and Nonpolar Media. J. AM. Chem. Soc. 2012, 134 (45), 18585-18590.

3. VanOrman, Z. A.; Bieber, A. S.; Wieghold, S.; Nienhaus, L., Green-to-Blue Triplet Fusion Upconversion Sensitized by Anisotropic CdSe Nanoplatelets. Chem. Mater. 2020, 32 (11), 4734-4742.

4. Beyler, A. P. Single-nanocrystal photon correlation: a versatile tool for elucidating basic physics and characterizing applications-relevant properties. Massachusetts Institute of Technology, 2015.

5. Brokmann, X.; Bawendi, M.; Coolen, L.; Hermier, J. P., Photon-correlation Fourier spectroscopy. Opt. Express 2006, 14 (13), 6333-41.

6. Coolen, L.; Brokmann, X.; Spinicelli, P.; Hermier, J. P., Emission characterization of a single $\mathrm{CdSe}-\mathrm{ZnS}$ nanocrystal with high temporal and spectral resolution by photon-correlation Fourier spectroscopy. Phys. Rev. Lett. 2008, 100 (2), 027403.

7. Hu, Z.; Singh, A.; Goupalov, S. V.; Hollingsworth, J. A.; Htoon, H., Influence of morphology on the blinking mechanisms and the excitonic fine structure of single colloidal nanoplatelets. Nanoscale 2018, 10 (48), 22861-22870.

8. Marshall, L. F. Spectral dynamics of single quantum dots: A study using photon-correlation Fourier spectroscopy for submillisecond time resolution at low temperature and in solution. Massachusetts Institute of Technology, 2011.

9. Spokoyny, B. Photon Arrival Time Correlation, MATLAB Central File Exchange, 2017. 